\title{
La proyección social de la universidad y La salud para todos y por todos (actualización)
}

\author{
The social projection of university and Health for all and by all (up grade) \\ Hernán Málaga Cruz ${ }^{1}$
}

\section{RESUMEN}

El objetivo de la intervención fue aportar a la equidad en salud, desarrollando la comunidad saludable de los Jardines de Manchay. Para ello, los alumnos de los cursos de Salud Pública y de Responsabilidad Social de La Escuela de Ciencias Veterinarias de la Universidad Ricardo Palma, conjuntamente con la comunidad, seleccionaron seis problemas a intervenir. La metodología utilizada fue la lluvia de ideas, la priorización de los problemas, la encuesta a las familias y la venta de proyectos elaborados en marco lógico. Los resultados más importantes fueron el control de la anemia mediante el consumo de huevos y el mejoramiento de la calidad de vida en general de sus habitantes. En conclusión, ha quedado establecida una comunidad saludable con resultados de equidad, que hacen de ella una comunidad organizada para enfrentar problemas y buscar el bien común.

Palabras Claves: Salud Pública, Marco lógico, Comunidad saludable, Equidad.

\begin{abstract}
The objective of the intervention was to contribute to health equity, developing the healthy community of "Jardines de Manchay". For that, the students of the Public Health and Social Responsibility courses of the School of Veterinary Sciences of Ricardo Palma University, together with the community, selected six problems to intervene. The methodology used was brainstorming, problem prioritizing, surveying families and selling projects developed in a logical framework. The most important results were the control of anemia through the consumption of eggs and the improvement of the general quality of life of its inhabitants. In conclusion, a healthy community has been established with equity results, which made it an organized community to face up problems and seek the common good.
\end{abstract}

Key Words: Public Health, Logical Framework, Healthy community, Equity.

\section{INTRODUCCIÓN}

En 1978, representantes de todos los países del mundo, se reunieron en Alma Ata en la Ex Unión Soviética, para desarrollar una estrategia de atención primaria de salud como el camino para lograr la Salud para Todos en el año 2000. Esta idea fue concebida como la resolución de problemas esenciales de salud, con plena participación social, trabajo intersectorial, tecnologías apropiadas y a un costo que la propia comunidad pudiera solventar. El objetivo era lograr la equidad en salud y así disminuir las brechas existentes en los principales indicadores: mortalidad infantil, desnutrición crónica infantil, mortalidad materna $\mathrm{y}$ expectativa de vida (OMS, 1978).

En 1986, países desarrollados suscribieron la célebre Carta de Ottawa, en la que se describieron los prerrequisitos de la salud: paz, educación, empleo digno, seguridad alimentaria, vivienda y saneamiento básico, es decir, justicia social, caracterizada por la satisfacción de las necesidades para una vida digna,

Profesor de Salud Pública y Responsabilidad Social de la Escuela de Ciencias Veterinarias de la Universidad Ricardo Palma. 
(OMS, Ministerio de Salud y Bienestar Social de Canadá, Asociación Canadiense de Salud Pública, 1986). A todo lo expuesto, la Carta de Bogotá añade el acceso al bienestar (Restrepo y Málaga, 2001).

Los países de América Latina tienen grandes desigualdades en las determinantes de salud mencionadas, lo que, aunado a las inequidades en el acceso a servicios de salud de igual calidad ante una igual necesidad, crean las grandes diferencias en materia de salud, enfermedad y muerte de las poblaciones (Málaga, Dachs, Perdomo y Guemes, 2005).

Todas las inequidades existentes son injustas cuando son evitables y, por lo tanto, es deber del Estado y de la sociedad civil en su conjunto buscar alcanzar la misión de la salud pública: proporcionarle a la gente las condiciones para ser saludables (Mann, 1997). Desde los cursos de Salud Pública y de Responsabilidad Social de La Escuela de Ciencias Veterinarias de la Universidad Ricardo Palma (URP), se decidió enseñar la nueva salud pública, la que actúa sobre los factores determinantes de salud y enfermedad, interviniendo sobre factores de riesgo y promoviendo factores protectores. Como previamente se había enseñado a formular proyectos por el método del marco lógico, se propuso el plan siguiente para integrar a la Universidad en una solución al problema planteado:

a) Reconocer la existencia del problema, demostrando evidencias de una comunidad con exclusión social.

b) Hacer conciencia de la problemática en la comunidad a intervenir.

c) Intervenir la comunidad excluida para buscar la equidad en salud.

d) Difundir los resultados entre la comunidad universitaria, para conseguir la participación interdisciplinaria.

e) Divulgar la experiencia al gobierno nacional, al Ministerio de Salud, gobiernos locales, sociedad civil, organismos internacionales de cooperación en salud $y$ otros con responsabilidades en promoción de la salud.

\section{SELECCIÓN DE LA COMUNIDADA Y DIAGNOSTICO SITUACIONAL}

\section{PROCESO DE SELECCIÓN DE LA COMUNIDAD}

En el segundo semestre de 2012, con la primera promoción de la Escuela de Ciencias Veterinarias y con alumnos del curso de salud pública, se escogió al distrito de Pachacamac por su cercanía a la universidad y, porque en el Centro de Salud del distrito se analizó las condiciones de vida de cuatro comunidades dentro del mismo. Con esta información y las estadísticas de desnutrición crónica infantil notificadas a la unidad sanitaria, se encontró una correlación injusta entre la intensidad de la exclusión y la desnutrición crónica infantil, escogiéndose a la comunidad de Manchay por tener la mayor evidencia de exclusión social.

Ya en Manchay y con la venia de los presidentes de las juntas vecinales, en febrero de 2013 se escogió a la comunidad de Los Jardines de Manchay, a la que en adelante se denominará "Comunidad Saludable de Los Jardines de Manchay". Se demostró el compromiso de eliminar estas diferencias, expresadas por su presidenta, Ahida Nelly Denegri, y la comunidad en pleno. El compromiso se plasmó en el Proyecto de "Comunidad Saludable de Los Jardines de Manchay", que se presentara a la Oficina de Extensión Cultural y Proyección Social de la Facultad de Ciencias Biológicas de la URP.

\section{DESCRIPCIÓN DE LA COMUNIDAD SELECCIONADA}

La comunidad poseía 485 lotes habitados de 500 registrados, de las cuales 367 pertenecían al sector k y 122 al sector L. Contaba con aproximadamente 700 familias, y tenían en ese entonces un centro comunitario, el que se había construido en un lote de media manzana en el sector $\mathrm{k}$. El centro comunitario se reducía a un área techada y con base de cemento y un local cerrado, donde se contaba con una cocina. El comedor comunitario, aún sin construir, ocupaba un área en la entrada. Todo ello se había construido con aportes de la comunidad y actividades sociales.

\section{PRIORIZACIÓN DE PROBLEMAS}

Con la segunda promoción y por lluvia de ideas de la comunidad, se identificaron catorce problemas, a los que se les caracterizó según su importancia y posibilidades de solución a mediano plazo. Definiéndose así cinco problemas como prioritarios: seguridad alimentaria, seguridad ciudadana, embarazo de adolescentes y empleo juvenil, control de perros callejeros y carencia de áreas verdes y de recreación infantil. Así, se elaboraron los árboles de problema y los proyectos de intervención con la metodología de marco lógico en mesas de trabajo con la comunidad. Los mismos que se presentaron en la primera ceremonia de clausura de actividades, la que contó 
con la presencia del Dr. David Tejada de Rivero, ex Ministro de Salud del Perú, ex subdirector de la Organización Mundial de la Salud, y organizador y participante de Alma Ata, quien definió los problemas de salud a través de "la sabiduría natural de un viejo árabe: cinco enfermedades de los camellos, la falta de agua segura y tres enfermedades de la gente", postulando así la actualmente denominada "una salud: ambiental, animal y humana".

La tercera promoción realizó una encuesta a cien hogares mediante un muestreo estratificado proporcional a los estratos conformados por los sectores $1 \mathrm{y} \mathrm{k}$. Los resultados arrojaron las siguientes conclusiones: se halló una desnutrición crónica infantil del $5 \%$, con diferencias de género pues los niños están mejor nutridos que las niñas; el embarazo de adolescentes en un $8 \%$, la tasa de incidencia anual de mordeduras caninas en un $9 \%$ y una incidencia de un $4 \%$ de actos delincuenciales sobre sus habitantes. Un $27 \%$ de las familias había sembrado un árbol $(0.24 \leq \mathrm{p} \leq 0.30) 95 \%$ y solo en un $32 \%$ de los hogares los niños practicaban deporte $(0.32 \leq \mathrm{p} \leq 0.39) 95 \%$. (Málaga, Piña, Denegri, Stefan, Cedamanos, Alvarado et al., 2014).

\section{PROYECTOS DE INTERVENCIÓN}

\section{DESCRIPCIÓN DE LOS PROYECTOS}

A continuación se describen las intervenciones realizadas a través de proyectos y sub proyectos en la "Comunidad Saludable de Los Jardines de Manchay"

\section{Proyecto de Seguridad Alimentaria:}

Propósito: alcanzar la seguridad alimentaria en la población intervenida.

a. Sub-proyecto de Gallinas Ponedoras.- En el primer semestre del 2015 se consiguió un donativo de cinco mil dólares de la fundación benéfica Pro Niño, fondo que fue administrado por la URP. Los recursos permitieron comprar seiscientas pollitas de doble propósito y alimentarlas hasta la semana veinticinco, las que fueron repartidas en treinta familias con hijos menores de 5 años, quienes construyeron corrales para recibir las pollitas, previa capacitación por parte de los profesores Pablo Reyna y Jorge Sarria de la URP.

A este donativo se unió el Laboratorio Bang (representado por M.V. Rubén Pinedo), quien donó treinta comederos y bebederos lineales. Los alumnos visitaron además las casas antes de la recepción de las pollitas a fin de verificar las instalaciones en las que serían albergadas. Las vacunas contra Newcastle-Laringotraqueitis y Gumboro fueron donadas por el Laboratorio Farvet (representado por el M.V. Manolo Fernández), y la vacuna recombinada de viruela-bronquitis aviar fue donada por Laboratorio Ceva (por gestión del M.V. Roberto Valdivia). Ambas vacunas fueron aplicadas por alumnos del curso, y en enero de 2015 por alumnas voluntarias, quienes a su vez capacitaron a las beneficiarias para desarrollar esta actividad.

En el año 2016 se estableció la Cooperativa Virgen de Guadalupe para facilitar el expendio de los huevos, recibiendo asesoría de la Escuela de Administración y Gerencia, mediante los docentes Jorge Carbajal y Américo Soto, entre otros. Sin embargo, esta experiencia funcionó solamente por un periodo de tres meses, después de lo cual se desactivo dejando en libertad a los beneficiarios de utilizar su producción de manera independiente.

El impacto de esta intervención fue medido mediante una tesis de pre-grado que evidencia el control de la anemia infantil, la que se encontraba en $44 \%$ ( 8 de 18 niños menores de 5 años) en marzo del 2015 y que a través del consumo de 2 huevos diarios por los niños y por los familiares, se redujo a cero en 16 de ellos, examinados en diciembre de $2015(\mathrm{P}<0.01)$ (Muñoz, 2017).

Durante el desarrollo del proyecto entre el 06 de noviembre de 2014 al 18 de febrero de 2020, se han hecho 21 entregas a más de 119 familias diferentes, habiéndose entregado 6062 pollitas (Información actualizada en abril de 2020). En cada recepción de pollitas, los alumnos intervinieron vacunándolas y asesorando a los productores en ambientación, alimentación, manejo y sanidad a las mismas.

Gramogen, empresa donante de varios lotes, firmó un convenio marco con la universidad y se ofreció participar como cooperador de la comunidad, expresado a través de una carta de intención de su actual presidente el Sr. Guillermo Condori, el que aseguró el aprovisionamiento de pollitas en el periodo 2017-2020. El 12 de julio del 2017, por cortesía de Gramogen se entregó 90 pavitos a 20 familias (4 a 5 por familia) para su cría, venta y consumo en la navidad. El Sr. Álvaro Hintze se unió al programa en mayo del 2018 donando 360 gallinas de levante, las que se distribuyeron a 18 familias. 
En ocasión de la reciente pandemia de Covid 19, y ante la amenaza de acabar con el sistema productivo familiar establecido, por venta de gallinas y por escasez de alimentos, el benefactor Sr. Álvaro Hintz donó tres toneladas de alimentos, lo que hizo posible brindar 43 kilos de comida de postura para cada avicultor. La entrega se hizo en entre el 26 al 30 de 2020 de marzo por directivos de la comunidad y la egresada Srta. Katherine Mendoza representando a la universidad. En la primera ocasión, se respetó la distancia social para realizar el reparto y la gente acudía con sus respectivas mascarillas 2020 (Información actualizada en abril de 2020).

En este mismo proyecto, la ingeniera Berta Malqui, de la Escuela de Biología, realizó un curso de panadería para cinco señoras el año 2016.

b. Sub-Proyecto Crianza de cuyes.- Este proyecto fue concebido en el primer curso de responsabilidad social 2019-1, con la asesoría del M.V. Lucio Pando. En la primera reunión del mismo curso en el 2019-2, las representantes de la quinta promoción del Colegio Santa Rosa Maryknoll hicieron entrega al Dr Pando 2050 soles (Fondo Lucy Malaga) con lo que se comprarían 100 cuyes (80 hembras y 20 machos) con la finalidad de entregar 4 hembras y un macho a veinte familias escogidas por la comunidad. Antes de la entrega, las familias instalaron un área limitada por ladrillo de $1 \mathrm{~m}$ $\mathrm{x} 1.50 \mathrm{~m} \times 0.60 \mathrm{~m}$ de altura.

c. Sub-proyecto Comedor Comunitario.El Comedor, aún a medio construir, entró en funcionamiento mediante una donación de cincuenta raciones diarias hecha por la municipalidad de Pachacamac, las que eran preparadas por miembros de la comunidad. Además, se consiguió donaciones para el piso y mobiliario del comedor. Próximamente se ha de techar el comedor, pues la Municipalidad de Pachacamac ha donado el material de construcción para hacerlo.

\section{Proyecto Áreas Verdes y Recreación juvenil}

Propósito: aumentar las áreas verdes y lugares recreacionales.

a. Sub -Proyecto áreas verdes.- La cooperación del pastor Julio Piña permitió establecer en el primer semestre de 2017 una serie de biohuertos. En 2016, se capacitaron cinco familias por seis meses, con el objeto de obtener productos vegetales frescos, garantizando sus bondades nutritivas. A su vez, se consideró que ello podría representar una fuente de trabajo y distracción para las personas, enriqueciendo la vida de los agricultores en valores de respeto por el medio ambiente y reutilización de espacios abandonados o de poco uso para ser destinados al cultivo de hortalizas y verduras.

b. Sub -Proyecto de arte.- Se desarrolló un curso de flauta dulce, dirigido por la profesora Rosario Puñez de la Oficina Central de Extensión Cultural y Proyección Social (OCECPS), durante todos los sábados del primer semestre de 2016. Se realizaron además talleres de dibujo y pintura para niños menores a 8 años, para que exploten su creatividad. Se ha desarrollado un curso de guitarra por el $\mathrm{Sr}$. Germán Correa, amigo de la comunidad.

c. Sub -Proyecto Curso de Judo.- La Familia Marco Vigilato, Consultor Regional de PanAftosa, ha construido un tatami en la sede del Centro Comunitario, iniciándose con los amigos del Kami-judo un curso al que se inscribieron 60 niños y niñas de la comunidad. El 8 de abril de 2017 se realizó la bendición del Tatami por el Capellán de la Universidad Pbro. Francisco Chaverry. El curso se ha desarrollado durante todos los sábados de 2 a 4 pm y el 17 de junio de ese año, se entregaron 20 kimonos yudogui.

La capacitación en Judo, continua a pesar de que el M.V. Marco Vigilato fuera transferido a otro país, siendo sustituido por el Sensey Joel Moreno Lazarte y otros compañeros del Kami Judo Club. Desde marzo del 2018 es el Hitoshi Sport Center quien asesora y el 22 de diciembre del 2018, se graduaron los primeros judokas con cinturón blanco-amarillo y cinturón amarillo. El verano de 2019 fue aprovechado para ofrecer clases tres veces por semana.

\section{Proyecto sobre Embarazo de adolescentes}

Propósito: disminuir la frecuencia de embarazos de adolescentes en un 50 por ciento.

a. Sub-Proyecto adolecentes responsables.- Con la finalidad de que los jóvenes tengan conciencia y sepan responder con madurez a temas involucrados en la educación sexual, se realizaron las siguientes actividades: charlas de autoestima, respeto, madurez y estabilidad emocional, así como encuestas para conocer su nivel de conocimiento del tema y saber qué puntos necesitan 
ser reforzados. A las mesas de trabajo se ha acercado una madre adolescente, con lo que se muestra el impacto inicial de la propuesta.

Para el mejor uso del tiempo libre, se propuso el establecimiento de una biblioteca. Con este mismo propósito y el de formar a los jóvenes para el trabajo, en el segundo semestre de 2018, un grupo de estudiantes realizó un curso de grooming para canes, el que contenía capacitación para: lavado, peinado y corte de pelo. También se desarrolló dos cursos de cosmetología para las adolescentes.

b. Sub-proyecto Biblioteca Ricardo Palma.- La ceremonia de clausura en julio de 2015 tuvo la presencia del Rector de la URP, quien al escuchar el proyecto de biblioteca, entregó quinientos libros a la comunidad. La Biblioteca se instaló en el segundo piso del local comunal, encargándose los alumnos de donar tres mesas de lectura. Posteriormente, por donativos adicionales, se separaron los libros para niños de los de adolescentes y adultos. Luego se creó un sistema de identificación para los usuarios y se realizó un registro de usuarios. La Biblioteca funciona por las tardes, bajo los cuidados de la Srta. Rocío Lacma, quien previamente se capacitó en la Biblioteca de la Facultad de Ciencias Biológicas de la URP.

\section{Proyecto de Control de mordeduras canina}

Propósito: controlar la población de perros callejeros y disminuir en 50 por ciento los índices de accidentes por mordedura.

En abril de 2015, se adhirió al proyecto el curso de Instrumentación Quirúrgica de la Escuela de Ciencias Veterinarias de la URP. Los docentes y alumnos, con el propósito de disminuir la agresividad canina, operaron a siete perros a los que castraron, instalando tres quirófanos en el centro comunitario. Este operativo contó con la colaboración de Jeff Weber, M.V. norteamericano ganador de un premio Emmy, y los M.V. Úrsula Bezold, Mauricio Jara y Guillermo Risco, actuando los estudiantes como instrumentadores y a cargo de la UCI para la recuperación de los canes.

En otro semestre se castraron cinco perros machos y en posterior semestre, se castraron 9 perros machos. En posteriores castraciones los alumnos del curso de instrumentación, ejecutaron el proyecto y actuaron de cirujanos los alumnos de salud pública, quienes previamente identificaron a los perros machos, concientizaron a sus propietarios acerca de la importancia de la castración y una semana antes de la cirugía se les hizó un control de hemoglobina, hematocrito y un electrocardiograma, para declararlos aptos para la intervención. Actuaron como coordinadores de esta actividad los M.V. Alberto Delgado y Mauricio Jara y como anestesiólogo el M.V. Mario Pauta. En el segundo semestre del año 2018, se incluyó actividades de desparasitación de animales de compañía.

Para fijar la meta de castraciones, la alumna Gabriela Cristina Torres, como parte de su tesis, realizo el censo por muestreo de la población canina, estimándose esta en 615 perros, $65 \%$ de hogares con perro, 1,26 perros por casa, y $69 \%$ de los perros fueron machos, fijándose la meta de castraciones por la comunidad en ochenta perros. En el segundo semestre de 2018, no se pudo castrar ningún perro macho, por lo que se decidió cerrar este proceso, sin haber conseguido la meta.

También se ha realizado charlas informativas sobre beneficios de la castración en perros y gatos, parásitos externos e internos de las mascotas $\mathrm{y}$, con el propósito de que los perros no hociqueen la basura, se elaboró una canastilla modelo de hierro para el establecimiento de las basuras en alto. Hoy, estas canastillas y el buen uso se extienden por toda la comunidad.

\section{Proyecto Seguridad ciudadana}

Propósito: Disminuir la violencia familiar y comunitaria.

Con la asesoría de un voluntario, Sr. César Guerrero de Villa María del Triunfo, se puso en funcionamiento un sistema de alarmas y pitos en la comunidad, con las cuales, en el verano de 2016, se pudo capturar un delincuente en la madrugada. Se solicitó a la Empresa Luz del Sur la iluminación de la cancha multiusos para evitar la delincuencia juvenil durante las noches, lo que ha sido ya realizado, Luz del sur ha atendido a diferentes solicitudes de vecinos, gestionados por los alumnos; sin embargo, aún quedan zonas sin alumbrado público. El semestre 2019-2 se contó con la cooperación del Coronel (retirado) Pedro Tello del Ejército del Perú, quien fuera jefe de seguridad ciudadana de la municipalidad de Lima.

Este proyecto promovió actividades deportivas en la comunidad: campeonatos de vóley y fulbito. 
Para estimular el interés en esta área, la comunidad ha sido visitada por tres personajes del deporte: Raúl Leguía, Director Administrativo del Club Universitario de Deportes; su entrenador en ese momento Sr. Roberto Challe y el ex jugador Héctor Chumpitaz, conocido como el "Capitán de América".

Durante dos semestres se contó con la asesoría del coronel (retirado) de la Policía Raúl Campos Burgos.

\section{Proyectos Productivos}

Propósito: Desarrollo sostenible del Proceso.

Con el apoyo de la OCECPS se desarrolló un curso de cerámica por el profesor Dominino Vilca. El año 2017, la Fundación Benéfica Pro-Niño nuevamente apoyó el proceso, donando un horno eléctrico de marca HINSA, el que alcanzaba una temperatura de $1150^{\circ} \mathrm{C}$. Para el día de la madre, la Dirección de Recursos Humanos de la URP adquirió 100 floreros y maceteros, para las madres de la Universidad. El 8 de abril de 2017 se realizó la bendición del horno por el Capellán de la Universidad Pbro. Francisco Chaverry. El uso del horno ha quedado reglamentado y se ha preparado una página web para la venta por internet de las artesanías. Para el cuidado de las piezas producidas y su elaboración, se ha construido un pequeño taller de madera.

\section{PARTICIPACIÓN DE OTRAS ESCUELAS Y FACULTADES}

En el primer semestre de 2017, alumnos de las facultades de medicina y psicología y de la escuela de Biología, iniciaron su participación en algunos de los proyectos anteriormente descritos: embarazo de adolescentes y bio-huertos; y se amplió el proceso a control de desastres y cantinas escolares. De esta manera se empezaba a hacer realidad el trabajo intersectorial.

En el segundo semestre de 2018 se incluyó la propuesta Proyecto Salud Individual de las Personas, gracias a la MSC Dra. Darcy Guzmán Ángeles, el cual se inició con charlas de salud oral con miras a la instalación de un consultorio odontológico.

Así también, el segundo semestre de 2018, el silabo para el dictado de un curso Inter programático fue ofrecido a todas las escuelas y facultades de la universidad, previa aprobación por el consejo universitario. El curso recién se inició el primer semestre de 2019, con 4 estudiantes de veterinaria y dos estudiantes libres, entre ellas una profesional odontóloga; preparando 3 proyectos nuevos: Crianza de Cuyes, Parque Infantil y Consultorio Odontológico, este último próximo a implementarse el año 2020, habiéndose obtenido ya la aprobación del directorio de la Fundación Benéfica Pro-Niño. El segundo semestre del año 2019, y con 7 alumnos matriculados, se introdujo 3 proyectos nuevos: Cocina y Repostería, Higiene Personal y Plantas en el Hogar.

\section{DIVULGACIÓN Y DIFUSIÓN DE LA EXPERIENCIA Y TRABAJOS DE INVESTIGACIÓN PUBLICADOS}

A continuación se citan los medios por los que se ha divulgado y difundido la experiencia a fin de que sea tomado como un modelo que podría ser replicado en otras zonas vulnerables pero con espíritu de progreso.

I. Divulgación:

a. El Grupo de Facebook Comunidad saludable Los Jardines de Manchay tenía 689 miembros al 20 de abril de 2016. Al 19 de diciembre de 2018, tiene 2398 seguidores.

b. Se imprimieron mil trípticos para divulgación, con fondos de la Facultad de Ciencias Biológicas.

c. Presencia en la revista Propuesta Página web: https://hmalaga.wix.com/los-jardines-manchay

d. Entrevista y visita a la Comunidad por el Diario El Comercio, publicado el domingo 18 de marzo de 2018.

e. Entrevista y visita por el programa de TV Al sexto día el 28 de marzo de 2018.

f. Entrevista y visita por Televisa, México.

g. Entrevista del programa Comiendo con Causa de TV Perú en 2018.

h. Entrevista por el Diario El Comercio, al Dr. M.V. Hernán Málaga: "Las gallinas de los huevos de oro contra la anemia", publicada el domingo 15 de setiembre de 2019.

II. Difusión:

a. Revista de Ciencias Veterinarias desde 2012 (varios trabajos publicados).

b. Educación Veterinaria IV Reunión Científica APEMEV. 28 de agosto de 2015, UNMSM.

c. IV Foro Promoción de la Salud. La enseñanza de la nueva salud pública. 25 de noviembre de 2015 . Colegio Médico del Perú.

d. Jueves Técnico. La enseñanza de la nueva salud pública. Oficina Sanitaria Panamericana, 14 de enero de 2016. 
e. Municipalidad del Agustino, Conferencia: Comunidad Saludable los Jardines de Manchay, ante los Comités de seguridad ciudadana de diferentes Municipios de Lima Metropolitana, abril de 2016.

f. Entrevista realizada por Manuel Pantigoso sobre la Comunidad Saludable de Los Jardines de Manchay y Hora Segura en Lima Metropolitana. Radio Filarmonía, programa Palma Mater, 22 de junio de 2016.

g. Congreso de Promoción de la Salud. Trabajo aceptado para presentación en Congreso.22-26 de mayo de 2016. Curitiba, Brasil.

h. XXII Congreso Nacional de Ciencias Veterinarias. La enseñanza de la nueva salud pública. 7 de setiembre de 2016.

i. Municipalidad de Breña, Conferencia: Comunidad Saludable los Jardines de Manchay, ante la red de municipios y comunidades saludables de Lima Metropolitana. Setiembre de 2016.

j. Colegio Médico del Perú. Consejo Regional III-Lima: "Curso de Actualización en Gestión y Administración en Salud: Salud Pública en Gobiernos Locales". 1 de diciembre de 2016.

k. Universidad Peruana Unión. La Enseñanza de la Nueva salud Pública. Ante los Directores de Proyección Social de las diferentes facultades. UPU, 8 de marzo de 2017.

1. Universidad Alas Peruanas. Semana Internacional de la Universidad Alas Peruanas. La enseñanza de la nueva Salud Publica en Veterinaria.19 de mayo de 2017.

m. XI Curso -Taller Nacional de Atención Primaria en Salud Sociedad Científica Medico estudiantil. La enseñanza de la nueva salud pública. San Fernando 23 de febrero de 2018.

n. Sociedad Científica de Estudiantes de Medicina de la Universidad Ricardo Palma. Hablemos de salud pública. URP 10 de abril de 2018.

o. Curso de Salud Pública en Veterinaria. Maestría en Epidemiología y Salud Pública en Veterinaria. Expositor: Katherine Mendoza. "La enseñanza de la nueva salud pública”, 8 de junio de 2018.

p. Ministerio de Salud (MINSA). 1er Especio de dialogo para el fortalecimiento de las intervenciones de promoción de la salud. - La enseñanza de la nueva salud pública. 27 de junio de 2018 .

q. 9no.Congreso Iberoamericano de Universidades Promotoras de Salud, Monterrey 21-24 de mayo de 2019. La presentación del Proyecto por Katherine Mendoza consiguió el Primer Puesto en respuestas universitarias a los problemas de salud Pública.

\section{Trabajos de investigación publicados:}

a. Málaga H, Piña J, Denegri A, Stefan T, Cedamanos D, Alvarado D, et al. Priorización de problemas de salud comunitaria en una localidad de Lima Metropolitana en el marco del programa de Municipios Saludables. Rev peru epidemiol 2014; 18(2): e08.

b. Muñoz-Saldaña Y, Málaga H. Resumen Control de la anemia infantil, por el consumo de huevos de gallina. 5to Congreso Iberoamericano de Medicina Familiar y Comunitaria Hotel West Inn, Lima, Perú 17 de agosto 2017.

c. Muñoz-Saldaña Y, Málaga H. Control de la anemia en niños, mediante el consumo de huevos de gallina de traspatio. Revista Biotempo 14(2) Juliodiciembre 2017:159-165pp.

d. Málaga H. La Responsabilidad social de las empresas y personas jurídicas en el desarrollo social de las comunidades a través de la Universidad: El caso Universidad Ricardo Palma. Revista Tradición, Segunda época 2017 N¹7 pp51-56.

\section{CONCLUSIONES Y RECOMENDACIONES}

- La escogencia de comunidades excluidas se facilita por la correlación entre condiciones de vida y desnutrición crónica infantil.

- La selección de prioridades debe hacerse siempre junto a la comunidad, y una encuesta de hogares es un buen procedimiento para refrendar su escogencia.

- El uso del marco lógico para el diseño de los proyectos con participación comunitaria y asesoría de estudiantes es factible y recomendable.

- Las reuniones de venta de proyectos para financiar los mismos, en el que participen posibles benefactores, son muy recomendables.

- La presencia de líderes nacionales y locales contribuye a la credibilidad del proceso por la comunidad y por los estudiantes.

- La anemia infantil es controlable por el consumo de huevos de gallina.

- La participación de estudiantes y profesores de distintas profesiones los transforma en impulsores de la inclusión social y promueve el trabajo interdisciplinar

- El Curso de Salud Pública ha recibido hasta la fecha 15 Promociones y 14 de ellas han propiciado el desarrollo descrito anteriormente. El curso de Responsabilidad Social ha propuesto seis nuevos proyectos, dos de ellos en ejecución.

- La intervención comprueba que la salud de la comunidad es la resultante de la satisfacción de sus 
pre-requisitos, tal cual lo señala la carta de Ottawa: paz, educación, seguridad alimentaria, vivienda, empleo digno, saneamiento básico, justicia social y acceso al bienestar.

\section{REFERENCIAS BIBLIOGRAFICAS}

1. Mann, J. M. (1997). Medicine and public health, ethics and human rights. Hastings Center Report, 27(3), 6-13.

2. Málaga, H., Dachs, N., Perdomo, M., \& Guemes, A. (2005). Problemas Éticos de la Salud Pública. Rev. Acad. Peru. Cinc. Vet 5, 6, 21-32.

3. Málaga, H., Piña, J., Denegri, A., Stefan, T., Cedamanos, D., Alvarado, D., ... \& Santacruz, M. (2014). Priorización de problemas de salud comunitaria en una localidad de Lima Metropolitana en el marco del programa de Municipios Saludables. Revista Peruana de Epidemiología, 18(2), $1-4$.
4. Muñoz, Y. C. (2017). Control de la anemia en niños menores de 9 años por el consumo de huevos de gallina de traspatio en la comunidad saludable los Jardines de Manchay. Tesis de Título de Medica Veterinaria Escuela de Ciencias Veterinarias, Universidad Ricardo Palma.

5. Organización Mundial de la Salud, Ministerio de Salud y Bienestar Social de Canadá, Asociación Canadiense de Salud Pública. (1986). Carta de Ottawa para la Promoción de la Salud, Ottawa, Canadá 17-21 noviembre.

6. [OMS]Organización Mundial de la Salud. (1978). Atención Primaria de Salud. Serie Salud para Todos $\mathrm{N}^{\circ} 1$, Ginebra.

7. Restrepo, H. E., \& Málaga, H. (2001). Promoción de la salud: cómo construir vida saludable. Pan American Health Org. Editorial Médica Panamericana, Bogotá, 298 pp. 